\title{
Successful treatment of acutely occluded left main coronary artery in a patient with an aortic valve bioprosthesis
}

\author{
Aneta Fronczak ${ }^{1}$, Jerzy Pręgowski ${ }^{1}$, Paweł Tyczyński ${ }^{1}$, Mariusz Truszczyński ${ }^{2}$ \\ ${ }^{1}$ Department of Interventional Cardiology and Angiology, Institute of Cardiology, Warsaw, Poland \\ ${ }^{2}$ Department of Interventional Cardiology, District Hospital, Leszno, Poland
}

Postep Kardiol Inter 2015; 11, 4 (42): 330-333

DOI: $10.5114 /$ pwki.2015.55606

\section{Introduction}

Flow-limiting left main coronary artery (LMCA) thrombus is a life-threatening condition, unless immediate and successful treatment is undertaken.

We present a case of a patient with large thrombus formation within the LMCA, which occurred one day after diagnostic coronary angiography.

\section{Case report}

The 63-year-old male patient was hospitalized due to non-ST elevation myocardial infarction. His previous history included aortic valve replacement with bioprosthesis implantation and saphenous vein graft (SVG) bypass to the right coronary artery (RCA) 2 years ago, diabetes mellitus type II, previous thrombophlebitis and arterial hypertension.

The current pharmacological treatment included bisoprolol, angiotensin-converting enzyme inhibitors (ACE-I), aspirin and statin. A loading dose $(600 \mathrm{mg})$ of clopidogrel was administered before admission to the hospital.

Echocardiography examination showed a well-functioning aortic bioprosthesis, preserved left ventricle ejection fraction and mild left ventricle wall hypertrophy.

Troponin level on admission was slightly elevated to $0.11 \mathrm{ng} / \mathrm{ml}$ (ULN $0.014 \mathrm{ng} / \mathrm{ml})$.

The angiography demonstrated trifurcation of the LMCA with borderline lesions in the left coronary artery (Figures $1 \mathrm{~A}, \mathrm{~B}$ ), and a borderline lesion in the proximal segment of the RCA with competitive flow to its distal segment from both the native RCA and patent SVG (Figures $1 \mathrm{C}, \mathrm{D})$.

One day after the catheterization and just before planned discharge, the patient developed acute chest pain with signs of cardiogenic shock. ECG showed ST-segment elevation in I, aVL, aVR and V4-V6 leads with concomitant right bundle branch block and left anterior hemiblock (Figure $2 \mathrm{E}$ ).

Immediate (within $10 \mathrm{~min}$ ), repeated angiography revealed a large thrombus within the LMCA, almost totally filling the lumen with TIMI 2 flow downstream in the main epicardial arteries (Figures 2 A, B). Abciximab and a loading dose of ticagrelor were administered. Despite several passages of manual thrombectomy, LMCA patency was not improved. Thus, percutaneous coronary intervention (PCI) with implantation of two everolimus-eluting stents (3.5 $\times 20 \mathrm{~mm}$ and $4.0 \times 12 \mathrm{~mm}$ ) into the LMCA and ostium of the intermediate branch $(\operatorname{InB})$ was performed. A good angiographic result in the LMCA was achieved. The patient's haemodynamic status improved and ECG normalized (Figure 2 F). Taking into account the given contrast amount and stabilized course, the borderline ostial lesion in the left anterior descending coronary artery (LAD) was left untreated. This lesion was visualized again in control angiography 3 days later and assessed as significant. Thus, same-time $\mathrm{PCl}$ with a radial approach was performed. After predilatation of the ostia of the LAD and left circumflex coronary artery (LCX), a $3.5 \times 18 \mathrm{~mm}$ sirolimus-eluting stent at $14 \mathrm{~atm}$ was implanted into the LAD ostium. Finally sequential kissing balloon postdilatation was performed in the LAD/ $\operatorname{lnB}(3.5 \mathrm{~mm}$ and $3.5 \mathrm{~mm}$ at $12 \mathrm{~atm})$, LCX postdilatation (3.0 $\mathrm{mm}$ at $12 \mathrm{~atm}$ ), LAD/InB kissing re-postdilatation and LMCA postdilatation with $3.5 \mathrm{~mm}$ at $18 \mathrm{~atm}$ (Figures $2 \mathrm{H}-\mathrm{I}$ ). A good angiographic result was achieved (Figure $2 \mathrm{~J}$ ). A maximal troponin T increase to $1250 \mathrm{ng} / \mathrm{ml}$ was noted.

Transesophageal echocardiography performed subsequently did not show any thrombotic material in the Valsalva sinus.

\section{Corresponding author:}

Paweł Tyczyński MD, PhD, Department of Interventional Cardiology and Angiology, Institute of Cardiology, 42 Alpejska St, 04-628 Warsaw, Poland, e-mail: medykpol@wp.pl

Received: 19.01.2015, accepted: 15.03.2015. 

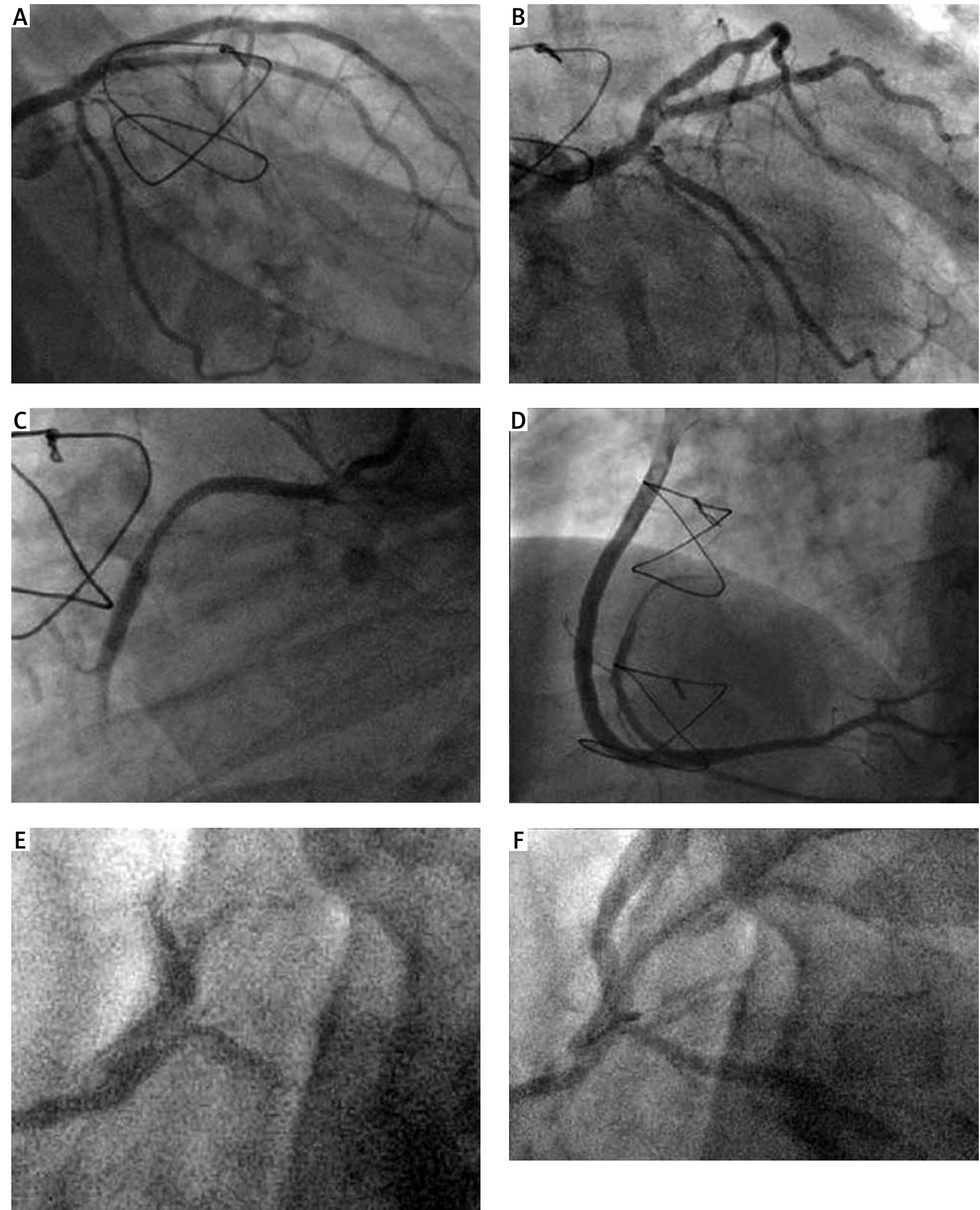

Figure 1. A, B - Left coronary artery without significant lesions. C, D - Borderline lesion in proximal segment of the right coronary artery with distal concomitant filling from both native artery and patent saphenous vein graft. E, F-Sequential images of non-coaxial intubation of the left main coronary artery 


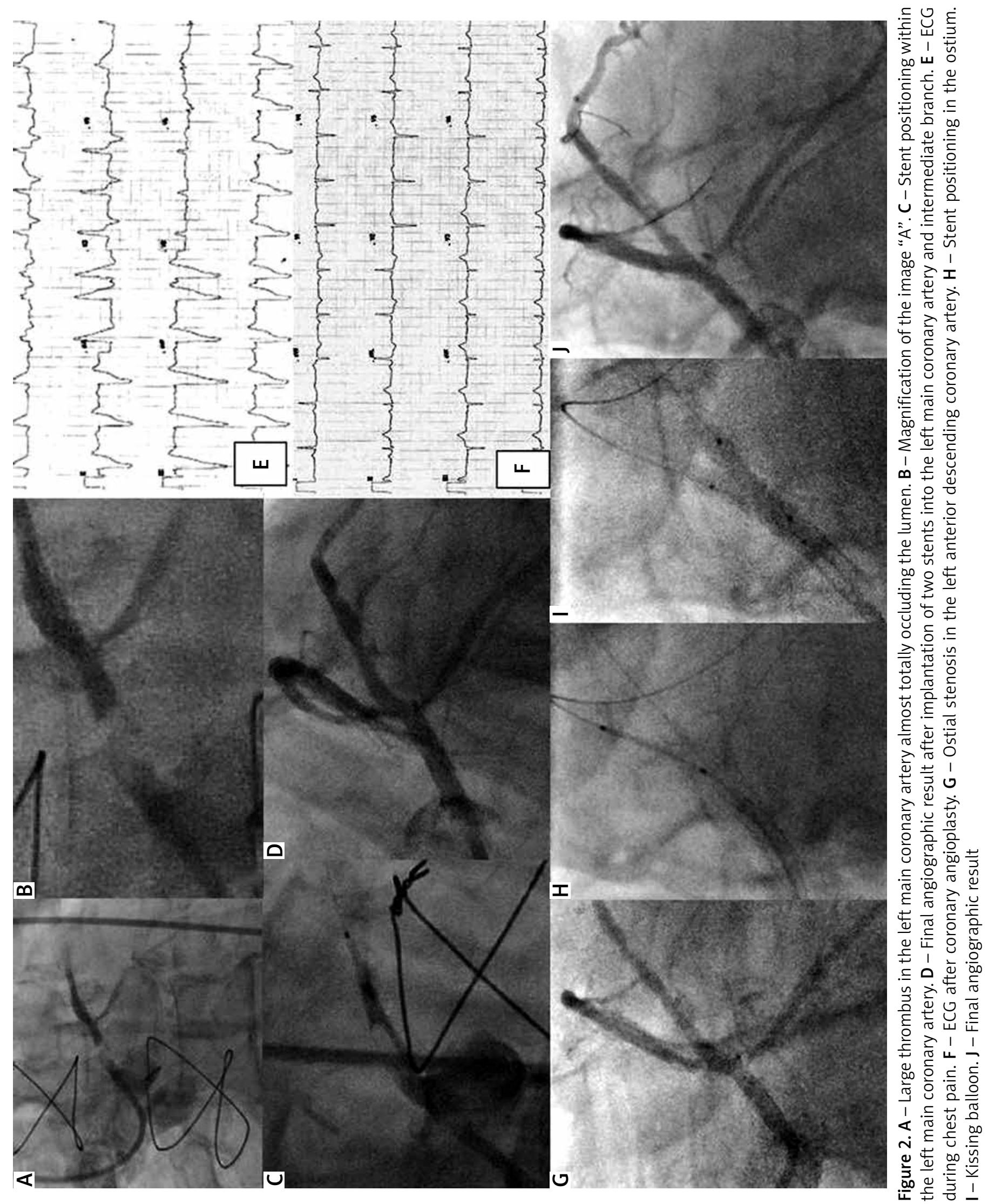


Double antiplatelet treatment (aspirin and ticagrelor) was prescribed for 12 months.

\section{Discussion}

The true incidence of LMCA thrombus remains unknown, as many of the patients die before reaching the hospital. The origin of LMCA thrombus in this case remains unclear. However, the close time-course correlation with angiography indicates two most likely event explanations. Firstly, angiographically non-visible catheter-induced LMCA dissection might have led to thrombus formation or subintimal hematoma propagation. Secondly, bioprosthetic-associated thrombus mobilization and migration into the LMCA as a complication of the angiography cannot be ruled out.

Non-coaxial LMCA intubation was seen in our case (Figures $1 \mathrm{E}, \mathrm{F}$ ), but no traces of any LMCA dissection were visible at the end of LMCA intubation. What is not seen in the angiography may be well visualized by optical coherence tomography (OCT). The OCT-based studies of the fate of stent implantation-induced edge dissections indicate that this is usually a benign phenomenon. Severe catheter-induced LMCA dissection is a rare and dramatic complication, and LMCA lumen compromise can happen immediately thereafter. Our patient did well nearly $24 \mathrm{~h}$ following diagnostic catheterization. Bioprosthetic valve-associated thromboembolism is a much rarer cause of acute coronary syndrome (ACS) compared to mechanical valves [1]. The ESC guidelines do not recommend anticoagulation therapy after aortic bioprosthetic valve implantation. This is supported by observations from the ANSWER registry, where the patients taking warfarin had a similar incidence of overall thromboembolism as those not taking warfarin [2].

Whatever the source, the consequences of coronary thromboembolism depend on the location and lumen compromise. Already the worst scenario had presented in our case, and only quick restoration of normal blood flow guaranteed the therapeutic success. In a study by Luca et al., among 24 patients with ACS due to thrombotic LMCA occlusion, the in-hospital mortality was as high as $58 \%$, and $80 \%$ ended in cardiogenic shock [3]. The therapeutic options include fibrinolysis, balloon angioplasty, stenting and adjunctive pharmacotherapy such as Ilb/Illa inhibitors. Some authors even suggest a Fogarty maneuver [4], but distal embolization is a risk. Thus, the appropriate treatment choice remains a challenge.

The role of thrombectomy after ambivalent results of four main randomized trials (TAPAS, INFUSE-AMI, TASTE and TOTAL) is questioned, and the number of successfully managed LMCA thrombus reports with this tool is very limited [5].

Next, what made the stenting even more challenging in our case was the presence of LMCA trifurcation, although only the LMCA body was affected by the thrombus and not the trifurcation region. According to the modified Medina classification for trifurcation or quadrifurcation lesions [6], the thrombus in the LMCA trunk represented the "1,0,0,0" scheme. However, plaque shift after stent implantation from the LMCA into the InB during the first $\mathrm{PCl}$ changed the anatomical scenario to " $0,1,0,0$ ". This lesion (LAD ostium) seemed angiographically significant during control angiography. Still, one might question its significance and ask for assessment by fractional flow reserve. While embarking on re-PCl, an additional stent for the LAD ostium was required with obligatory kissing balloon postdilatation. When LMCA trifurcating branches are comparable in diameter, simultaneous triple balloon kissing balloon postdilatation may be the option. Although not applied in this case, intravascular ultrasound guidance seems to be justified in such procedures, as in bifurcating LMCA PCI [7].

\section{Conclusions}

Finally, taking into account the complex PCI of LMCA trifurcation in the settings of ST elevation myocardial infarction and concomitant diabetes mellitus, a more potent $\mathrm{P}_{2} \mathrm{Y}_{12}$ inhibitor (ticagrelor) may be preferred over clopidogrel.

\section{Conflict of interest}

The authors declare no conflict of interest.

\section{References}

1. Karakoyun S, Gürsoy MO, Kalçık M, et al. A case series of prosthetic heart valve thrombosis-derived coronary embolism. Turk Kardiyol Dern Ars 2014; 42: 467-71.

2. Brennan JM, Alexander KP, Wallace A, et al. Patterns of anticoagulation following bioprosthetic valve implantation: observations from ANSWER. J Heart Valve Dis 2012; 21: 78-87.

3. De Luca G, Suryapranata H, Thomas K, et al. Outcome in patients treated with primary angioplasty for acute myocardial infarction due to left main coronary artery occlusion. Am J Cardiol 2003; 91: 235-8.

4. Eggebrecht $H$, Baumgart D, Naber $C$, et al. Extraction of large intracoronary thrombus in acute myocardial infarction by percutaneous Fogarty maneuver: intentional abuse of a novel interventional device. Catheter Cardiovasc Interv 2002; 55: 228-32.

5. Hajek P, Alan D, Vejvoda J, et al. Treatment of a large left main coronary artery thrombus by aspiration thrombectomy. J Thromb Thrombolysis 2009; 27: 352-4.

6. Tyczyński P, Karcz MA, Łazarczyk H, et al. Quadrifurcation of the left main coronary artery and acute coronary syndrome. Kardiol Pol 2015; 73: 299.

7. Can MM, Tanboga H, Karabay CY, et al. The treatment of acute myocardial infarction due to the occlusion of the left main coronary disease. Cardiol J 2011; 18: 77-82. 